\title{
Instrumentos para evaluar las habilidades motoras en niños con Trastorno del Espectro Autista entre 5 y 12 años: Revisión Sistemática Instruments to assess motor skills in children with Autism Spectrum Disorder between 5 and 12 years old: Systematic Review \\ Miriam Mariño Sánchez, Javier Rico Díaz, José Eugenio Rodríguez Fernández, Lucía Peixoto Pino U niversidad de Santiago de Compostela (España)
}

\begin{abstract}
Resumen. La actividad física es un elemento determinante en el desarrollo integral de la población. Por lo general, el al umnado conTEA esfísicamente inactivo como consecuencia, entre otros aspectos, de los déficitsmotrices existentes, lo que acaba perjudicando su desar rollo psicomotor. El objetivo de esta revisión sistemática es identificar y analizar los principales instrumentos utilizados para evaluar la motricidad en niños de entre cinco y 12 años diagnosticados conTEA. Se han revisado artícul os publicados entre los años 2015 y 2020, disponibles en cinco bases de datos electrónicas: PsycINFO, Web of Science, Scopus, SPO RTD Discusy PubM ed, siguiendo las pautas deladeclaración PRISM A pararevisiones sistemáticas. Tras laaplicación del método de búsqueday de los criterios de inclusión determinados, se analizaron en detalle un total de 10 documentos, en los que se refleja que los instrumentos más utilizados para evaluar el comportamiento motor de niños con autismo han sido las baterías o test, siendo laBatería MABC-2 la más completaentrelas que mejor se podrían adaptar para su uso en el contexto escolar. Se sugiere la elaboración de pictogramas que faciliten la comprensión de las pruebas por parte de los niños conTEA. Dada la importancia de mejorar sus habilidades motoras para facilitarles la práctica físico-deportiva, resulta determinante profundizar en el estudio de métodos de evaluación de su nivel motriz como punto de partida y de seguimiento para implementar intervenciones eficaces para esta población en el ámbito escolar.
\end{abstract}

Palabras clave. Trastorno del espectro autista, habilidades motoras, evaluación, instrumentos, niños.

\begin{abstract}
Physical activity is a determining element in the integral development of the population. In general, students with ASD are physically inactive consequently, among other things, of existing motor deficits, which ends up impairing their psychomotor development. The objective of this systematic review is to identify and analyze the main instruments used to assess motor skills in boys and girls between five and 12 years old diagnosed withASD. Articles published between 2015 and 2020, available in five electronic databases: PsycINFO, Web of Science, Scopus, SPO RTDiscus and PubM ed, have been reviewed, following the guidelines of the PRISMA statement for systematic reviews PRISMA methodology. After applying the search method and the deter mined inclusion criteria, atotal of 10 documents wereanalyzed in detail, in which it's reflected that the instruments most used to evaluate the motor behavior of boys and girls with autism have been batteries or test, being the MABC-2 Battery the most complete among those that could best be adapted for use in the school context. The development of pictogramsis suggested to facilitatetheunder standing of thetests by boys and girls withASD. It's important to improvetheir motor skills to facilitate physical-sporting practice, so it's essential to study in depth the methods to assess their motor level as a starting point and follow-up to implement effective interventions for this population in the school environment.
\end{abstract}

Key words. Autism spectrum disorder, motor skills, assessment, instrument, children.

\section{Introducción}

El Trastorno del Espectro Autista (TEA) es definido por el DSM - V, el Manual de losTrastornos M entales vigente, como un trastorno de neurodesarrollo, caracteriza do por manifestarse de diversas formas en función de su grado, del nivel de desarrollo y de la edad; de ahí el nombre espectro (American Psychiatric Association, 2014; García-Tabuenca, 2016). Fuentes, Hervás, \& Howlin (2020) destacan la importancia de una detección precoz

Fecha recepción: 31-01-21. Fecha de aceptación: 31-03-21

Javier Rico Díaz

javier.rico.diaz@usc del autismo en los primeros años de vida, en la que familias y docentes pueden jugar un papel determinante, comunicándole a los pediatras si observan demoras 0 anomalías tempranas relacionadas con el TEA. No obstante, Prieto, Cueto, Carballo-Fazanes, \& A belairasGómez (2020) ponen de manifiesto las limitaciones de las herramientas para la detección de posibles trastornos del desar rollo en edades tempranas por parte de los padres, no sólo en niños con TEA sino en la población infantil en general.

Los principales déficits que permiten establecer un diagnóstico deTEA son aquellos que están relacionados con el área de comunicación e interacción social (Reinders, Branco, W right, Fietcher, \& Bryden, 2019) 
y con el área de comportamientos restrictivos y repetitivos e intereses fijos (Adler, Minshawi, \& Erickson, 2014; APA, 2014; De Clercq, Aelterman, De Pauw, De Bolle, Decuyper, \& Tackett, 2010). EI TEA abarca una gran heterogeneidad de síntomas, destacando también deficiencias a nivel intelectual y/ 0 de lenguaje y a nivel motor (APA, 2014; Bishop, 2014; MacD onald \& Lord, 2011).

Los estudios que analizan el rendimiento motor de los niños con TEA reportan deficiencias en funciones como la destreza manual, el equilibrio, el control de objetosy habilidades locomotoras (M ache \&Todd, 2016). También se señalan dificultades en los patrones de la marcha, lo que genera «longitud de paso más corta, asimetría postural y dificultades para ejecutar el patrón de talón a punta» (Liu, 2013, p. 199). Estos déficits conllevan a una limitada participación en la actividad física $(A F)$, a pesar de ser necesaria para obtener un estilo de vida activo y saludable con el que prevenir el riesgo de sufrir enfermedades (Latorre-Román, Sánchez-Salvador, Salas-Sánchez, \& García-Pinillos, 2019; Ruiz-Vicente, et al., 2015; Sowa \& M eulenbroek, 2012), existiendo una mayor prevalencia de obesidad en los niños conTEA con respecto a la población en general (Kamal, Hanim, \& Ismail, 2019), así como una pérdida de autonomía personal (Reinders, et al., 2019).

Para contribuir a que los niños conTEA sean partícipes de las actividades físicas es necesario proporcionarles apoyo específico acorde a sus necesidades (Yu, Wong, Lo, So, \& Chan, 2018) a partir deunaenseñanzainclusiva, en la que estos niños, considerado un grupo de riesgo con respecto a la práctica de laAF, reciban una atención personalizada (Abarca-Sos, Murillo-Pardo, Julián-Clemente, Zaragoza-Casterad, \& Generelo-Lanaspa, 2015; Aragunde-Rodríguez, Rodríguez-Fernández, Fontenla Fariña, Gutiérrez-Sas, \& Pazos-Couto, 2018).

El movimiento corporal es muy importante para la vida humana, por lo que el trabajo y desarrollo de las habilidades motoras debe realizarse desde la infancia (Astorino, Contini, Fessia, \& Manni, 2018; García-Marín \& Fernández-López, 2020). En estesentido, López-Díaz, Moreno-Rodríguez, \& López-Bastías (2021), tras la rea lización de un programa deportivo específico con niños de entre seis y 12 años conTEA, reportan que éstos han logrado una mejora generalizada sobre las habilidades motrices y laAF, con un aumento eficaz en la capacidad de movimiento y de interacción con el entorno. Para conseguir que esas intervenciones sean eficaces, es fundamental analizar el punto de partida, a partir del cual se pueda obtener la información necesaria con la que orientar y enfocar las decisiones o acciones que sean pertinentes.

Por todo ello, el objetivo de esta revisión sistemática es identificar y analizar los principales instrumentos utilizados para evaluar la motricidad en niños entre cinco y 12 años diagnosticados con TEA y que, particularmente, se puedan aplicar al contexto escolar.

\section{Método}

El diseño utilizado en este estudio se ha basado en la revisión sistemática, siguiendo las pautas de la declaración PRISM A, que consiste en una guía dividida en fases con el objetivo de real izar revisiones sistemáticasy meta análisis con la mayor transparencia científica posible (Liberati, et al., 2009).

\section{Estrategia de búsqueda}

El proceso sistemático de búsqueda se llevó a cabo entre los meses de abril y mayo del año 2020 y para ello, se utilizaron cinco bases de datos internacionales: PsycINFO, Web of Science (WOS), PubMed, SPO RTDiscus y Scopus.

Las estrategias de búsqueda (tabla 1) incluyeron cua tro grupos de palabras clave (TEA, habilidades motoras, medición y población) y dos criterios de elegibilidad (idioma -sólo documentos en lengua castellana e inglés- y tipo de documento -artículo de revista-). Tras la aplicación de estos, y con el fin de obtener unos resultados aún más precisos, se empleó también el criterio de la fecha de publicación, incluyendo sólo documentos publicados en los últimos cinco años (2015 al 2020). Durante la indagación, se combinaron los distintos grupos de palabras, primero, através del conector booleano «OR»y, posteriormente con el conector «AND», para la selección de los artículos pertinentes para el análisis detallado.

\footnotetext{
Tabla 1

Estrategia de búsqued

\begin{tabular}{lll} 
Estrategia de busqueda \\
\hline 1.TEA Ti(autism OR autism spectrum disorder OR ASD) OR ab(autism OR autism
\end{tabular} spectrum disorder ORASD spectrum disorder OR ASD

\begin{tabular}{ll}
\hline 2. & Ti(motor skills OR motor development OR motor ability OR motor \\
Habilidades & competence) OR ab(motor skills OR motor development OR motor ability OR \\
motoras & motor competence)
\end{tabular}

3. Medición Ti(motor assess* OR motor instrument OR motor eval uat* OR motor test*) OR ab(motor assess* OR motor instrument OR motor evaluat* OR motor test*)

4. Población Ti(primary educat* OR primary school $O R$ elementar* school $O R$ child) $O R$ ab(primary educat* OR primary school OR elementar* school OR child)

ab(primary educat* $O R$ primary school $0 R$ elementar* schoo
$5.1,2,3$ y 4 limitadas a inglés 0 español y a artículo de revista o revisión

6. Combinación de las 4 ecuaciones (1 AND 2AND 3AND 4)

Ti:Title; Ab: Abstract
}

\section{Criterios de inclusión}

Una vez realizada la búsqueda en las bases de datos se procedió a la elección de los artículos en función de los criterios de inclusión establecidos. Estosúltimosfue- 
ron lossiguientes: 1) participantes con un rango de edad entre cinco y 12 años; 2) estudios que evaluaban las ha bilidades motoras y explicaban el instrumento utiliza do; 3) aplicación de la evaluación en un ámbito escolar 0 extraescolar. Teniendo en cuenta estos aspectos, se excluyeron todos los documentos sobre estudios que se llevaron a cabo en un laboratorio o entorno clínico, la edad de los niños se encontraba fuera del rango requerido 0 el objeto de análisis no era la evaluación de las habilidades motoras.

\section{Resultados}

\section{Resumen de los estudios incluidos}

En la búsqueda inicial se identificaron un total de 813 artículos. De ellos, se eliminaron 237 duplicados y se llevó a cabo un primer cribado de los 576 restantes a partir del título y los resúmenes, para descartar aquellos artículos que fuesen irrelevantes para la temática a estudiar. Del análisis de estos últimos, se excluyeron 551, quedando 25 textos paraunalecturaíntegraal cumplir todos ellos con los criterios de inclusión establecidos. Posteriormente, se excluyeron 15 de ellos, pues tras su lectura, se observó que tampoco cumplían con al guno de los criterios de inclusión. Finalmente, se incluyeron en esta revisión un total de 10 artículos (Figura 1).

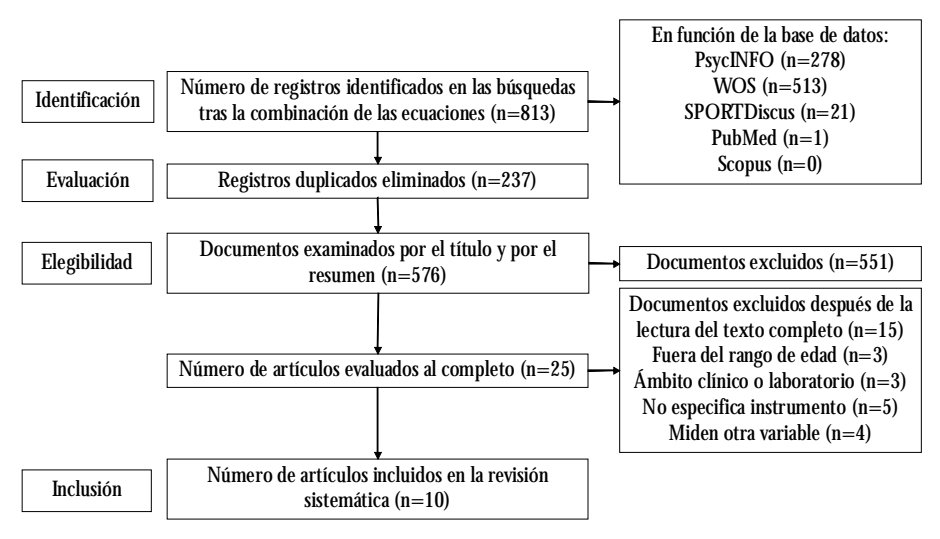

Figura 1. Diagrama de la selección de los artículos

\section{Características de los estudios}

En la tabla 2 se presenta, por orden alfabética de los autores, una síntesis de los 10 estudios que cumplían con los criterios de inclusión, sumando una muestra total de 1327 participantes, de los cuales, 483 fueron niños con autismo. Los restantes, eran niños/ as con desa rrollo típico, trastorno de coordinación yTDAH con los cuales se realizaron comparaciones en algunas de las investigaciones.
El tamaño de la muestra de los diferentes artículos de esta revisión se caracterizó por ser dispar y heterogéneo. Salvo uno de los estudios que contaba con una muestra amplia ( $n=1034)$, los restantes artículos disponen de un número más limitado. De estos nueve, siete cuentan con muestras de entre 14 y 49 integrantes, y los otros dos, estudios de caso, están compuestos únicamente por un participante en cada uno, uno de cinco y otro de nueve años.

La edad de los participantes en estas investigaciones oscilaba entre los cuatro y los 16 años.

Con respecto al diagnóstico TEA, dos artículos especificaron la participación de niños/ as con bajo coeficiente intelectual $(<70)$; seis documentos hicieron uso de muestras con alto funcionamiento ( $>70)$; y los dos restantes no especificaron grado de severidad.

\section{Discusión}

Existen deficiencias motoras en los niños con TEA, particularmente en funciones como la destreza manual, el equilibrio, el control de objetos y las habilidades locomotoras (Mache \&Todd, 2016). Como consecuencia, esto dificulta que puedan adquirir un hábito de práctica físico-deportiva regular y casi imposibilita que la puedan realizar deformainclusiva, con otrosniños (Astorino, et al . , 2018; M ache\&Todd, 2016; Reinders, et al. , 2019; Ruiz-Vicente, et al., 2015).

Con esta revisión sistemática, se pretendió identificar aquellos instrumentos que se ha demostrado que sirven para eval uar la motricidad en los niños conTEA, y que, particularmente, se puedan aplicar al contexto escolar.

Los instrumentos para evaluar las habilida des motoras que se han utilizado en las investigaciones de los artículos incluidos en esta revisión son principalmente baterías de pruebas 0 test, y permiten valorar diferentes destrezas, gruesasy finas, de los niños con dicho trastorno. Ú nicamente en uno de los estudios han utiliza do cuestionarios.

\section{Baterías o test}

Este tipo de instrumento es el más utilizado en los estudios de esta revisión para la medición de las habilidades motrices, gruesas y finas, de los niños con TEA. Destacan siete herramientas: The Bruininks-0 seretsky Test of Motor Proficiency (BOTMP), The Bruininks0 seretsky Test of Motor Proficiency-2 (BOT-2) y la versión corta (BOT-SF), M ovementAssessment Battery 
Tabla2.

Síntesis de los estudios que incl uyeron instrumentos para eval uar las habilidades motoras en escolares con TEA.

\begin{tabular}{|c|c|c|c|c|c|c|c|c|}
\hline Autor y & Título & Muestra & Diseño, intervención & O bjetivo & & Medición & Resultados & Limitaciones \\
\hline fecha & & & & & Instrumento & Destrezas evaluadas & & \\
\hline $\begin{array}{l}\text { Allen, } \\
\text { Bredero, }\end{array}$ & $\begin{array}{l}\text { Test of Gross } \\
\text { Motor }\end{array}$ & $\begin{array}{l}14 \text { niños con } \\
\text { TEA y } 21 \text { niños }\end{array}$ & $\begin{array}{l}\text { La evaluación se aplicó a } \\
\text { s niños con TEA durante } 2 \text { día }\end{array}$ & $\begin{array}{l}\text { Medir validez y } \\
\text { asconfiabilidad de la }\end{array}$ & $\begin{array}{l}\text { Test of Gross M otor } \\
\text { Development-3 }\end{array}$ & $\begin{array}{l}\text { Locomoción: carre } \\
\text { sal to, sal to horizont }\end{array}$ & $\begin{array}{l}\text { Se obtuvieron niveles } \\
\text { aceptables en las subpruebas }\end{array}$ & $\begin{array}{l}\text { Pequeño tamaño de la } \\
\text { s muestra; gran cantidad }\end{array}$ \\
\hline & Development-3 & de desar rollo & consecutivos. Se incluyó el & Prueba de desarrollo & & desl izamiento. & de las habilidades & de texto en las \\
\hline amme, & (TGMD-3) with & típico, entre 4 y & y protocolo tradicional & motor grueso-3, & & Con pelota: golpe de dos manos, & locomotoras y de & nuso exces \\
\hline rich, \& & de Use ofVisual & 10 años. & (instrucciones físicas y & para eval uar & & lpe de derecha con una mano, & el rendimiento $\mathrm{m}$ & imágenes; aus \\
\hline mons, & Supports for & & verbales); e un soporte vis & alrendimiento motor & & e, patada, tiro por & en los niños conTEA. Las & un largo perio \\
\hline 17. & $\begin{array}{l}\text { Children with } \\
\text { Autism Spectrum }\end{array}$ & & $\begin{array}{l}\text { (tarjetas con imágenes y } \\
\text { demostraciones). }\end{array}$ & $\begin{array}{l}\text { bruto de niños con } \\
\text { TEA. }\end{array}$ & & debajo con la mano. & $\begin{array}{l}\text { puntuaciones máximas del } \\
\text { grupo conTEA se alcanzaron }\end{array}$ & $\begin{array}{l}\text { adaptación; no se tuvo } \\
\text { nen cuenta el nivel de C }\end{array}$ \\
\hline & $\begin{array}{l}\text { Disorder:Validity } \\
\text { and Reliability. }\end{array}$ & & & & & & $\begin{array}{l}\text { con el apoyo del método } \\
\text { visual. }\end{array}$ & $\begin{array}{l}\text { función cognitiva o de } \\
\text { comunicación de los } \\
\text { participantes conTEA. }\end{array}$ \\
\hline & & & Informe de caso con un & Mejorar las & Test of Gross M otor & Habilidad & & Muestra de un solo \\
\hline olub- & $\begin{array}{l}\text { overhand } \\
\text { throwing Skill }\end{array}$ & $\begin{array}{l}\text { conTEA que } \\
\text { cursa } 30 \text { en una }\end{array}$ & $\begin{array}{l}\text { enfoque interdi } \\
\text { Intervención de }\end{array}$ & habilidades mo & Development-2 & mano por encima de la cabeza. & edispues & $\operatorname{larg}$ \\
\hline ez, 2 & for a child with & escuela & a13 sesiones de PT (30 & capacidad de & The Bruininks- & & actividades de lanzamiento & intervención; \\
\hline & $\begin{array}{l}\text { autism with a } \\
\text { collaborative }\end{array}$ & pública. & $\begin{array}{l}\text { minutos) y } 75 \text { sesiones de } \\
\text { ABA (10 minutos). Proyecto }\end{array}$ & $\begin{array}{l}\text { lanzamiento de manc } \\
\text { o del niño y su }\end{array}$ & $\begin{array}{l}00 \text { ser etsky Test of M otor } \\
\text { Proficiency-2 (BOT-2) }\end{array}$ & & & $\begin{array}{l}\text { instrumento no del } \\
\text { todo apropiado para }\end{array}$ \\
\hline & $\begin{array}{l}\text { approach in } \\
\text { school-based } \\
\text { therapy. }\end{array}$ & & $\begin{array}{l}\text { motor/ práctica de tiro; } \\
\text { participación en el juego. }\end{array}$ & $\begin{array}{l}\text { participación en las } \\
\text { actividades } \\
\text { recreativas. }\end{array}$ & & & & \\
\hline गाना & The impact of & 30 niños de 8 a & Todos participaron en un & Investigar el efecto & The Bruininks- & subpruebas motoras gruesa & joras significativas en & equeño tamaño de la \\
\hline
\end{tabular}

Eequeño tamaño de

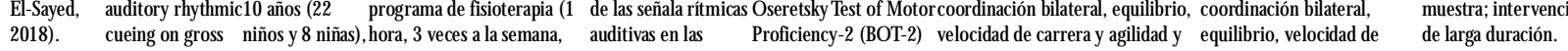

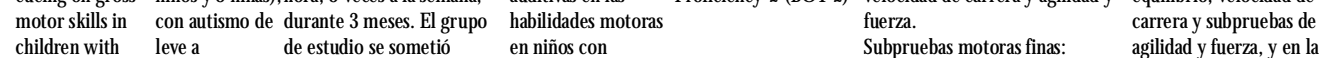
autism. moderado. 1 también a un entrenamiento autismo. precisión motora fina, integración coordinación corporal y grupo control y de marcha con Estimulación_ motorafinal, destreza manual y fuerza y agilidad, en ambos 1 grupo de RítmicaAuditiva d 30 $\begin{array}{lll}1 \text { grupo de } & \text { RitmicaAuditiva, d } 30 & \text { coordinacion de las extremidades grupos, pero con cambios } \\ \text { estudio. } & \text { minutos en } 3 \text { sesionesala } & \text { más destacados en el grupo }\end{array}$ semana, durante 3 meses. 


\begin{tabular}{|c|c|c|c|c|c|c|c|c|}
\hline $\begin{array}{l}\text { Mayes, } \\
\text { Breaux, } \\
\text { Calhoun, Frye, } \\
2017 .\end{array}$ & $\begin{array}{l}\text { High } \\
\text { prevalence of } \\
\text {, dysgraphia in } \\
\text { elementary } \\
\text { through high } \\
\text { school } \\
\text { students } \\
\text { with ADHD } \\
\text { and autism. }\end{array}$ & $\begin{array}{l}1034 \text { niños de } \\
f \text { entre los } 6 \text { y } 16 \\
\text { años con } \\
\text { inteligencia } \\
\text { normal }(C l=80) \\
\text { y diagnosticados } \\
\text { conTDAH } \\
\text { combinado } \\
(n=519) \text {, TDAH } \\
\text { de inatención ( } n= \\
227) 0 \text { autismo } \\
(n=288) .\end{array}$ & $\begin{array}{l}\text { La intervención se llevó } \\
\text { a cabo en los últimos } 20 \\
\text { años. En la pruebaVMI, } \\
\text { el participante debe } \\
\text { copiar formas } \\
\text { geométricas. Este } \\
\text { instrumento no tiene } \\
\text { tiempo y se cal ifica } \\
\text { utilizando criterios } \\
\text { estrictos (una regla y el } \\
\text { transportador para } \\
\text { medir longitudes, } \\
\text { proporciones y ángulos). }\end{array}$ & $\begin{array}{l}\text { Evaluar la prevalencia de la } \\
\text { disgrafía, utilizando el } \\
\text { mismo estándar de medidas } \\
\text { en un amplo rango de edad } \\
\text { (6-16) de estudiantes con } \\
\text { TDAH o autismo, con el fin } \\
\text { de determinar si la disgrafía } \\
\text { disminuye con los años. }\end{array}$ & $\begin{array}{l}\text { Developmental Test } \\
\text { ofVisual-M otor } \\
\text { Integration (VMI) }\end{array}$ & $\begin{array}{l}\text { Escritura de mano } \\
\text { (disgrafía) }\end{array}$ & $\begin{array}{l}\text { Más de la mitad de la } \\
\text { muestra total (59\%) } \\
\text { presentaba disgrafía y el } \\
92 \% \text { tenía dificultades en } \\
\text { la capacidad grafomotora } \\
\text { en relación con otras } \\
\text { habilidades. Además, la } \\
\text { frecuencia de disgrafía no } \\
\text { disminuye con la edad } \\
\text { entre los tres grupos } \\
\text { diagnósticos o edad. }\end{array}$ & $\begin{array}{l}\text { Participación solo de } \\
\text { niños con coeficiente } \\
\text { intelectual normal; } \\
\text { inter vención de extensa } \\
\text { duración; ausencia de una } \\
\text { evaluación formal de la } \\
\text { escritura a mano; } \\
\text { utilización de diferentes } \\
\text { versiones del test, por lo } \\
\text { que los resultados pueden } \\
\text { diferir. }\end{array}$ \\
\hline $\begin{array}{l}\text { Najafabadi, } \\
\text { Sheikh, } \\
\text { Hemayattalb, } \\
\text { Memari, } \\
\text { Aderyani, \& } \\
\text { Hafizi, 2018. }\end{array}$ & $\begin{array}{l}\text { The effect of } \\
\text { SPARK on } \\
\text { Social and } \\
\text { Motor Skills } \\
\text { of Children } \\
\text { with Autism. }\end{array}$ & $\begin{array}{l}28 \text { niños conTEA } \\
\text { de un rango de } \\
\text { edad de } 5-12 \text { años } \\
\text { y los cual es fueron } \\
\text { seleccionados al } \\
\text { azar y reclutados } \\
\text { de una clínica } \\
\text { terapéutica. Cabe } \\
\text { señalar el } \\
\text { abandono del } \\
\text { estudio de } 2 \\
\text { niños. }\end{array}$ & $\begin{array}{l}\text { Los niños fueron } \\
\text { divididos de forma } \\
\text { al eatoria en dos grupos: } \\
\text { tratamiento ( } n=12 \text { ), y } \\
\text { control ( } n=14) \text {. El } \\
\text { programa SPARK } \\
\text { consistió en } 36 \text { semanas } \\
\text { ( } 3 \text { sesiones por semana } \\
\text { de } 40 \text { min. de duración). } \\
\text { Los niños fueron } \\
\text { evaluados por BOTM P: } \\
\text { línea de base, antes de } \\
\text { comenzar y entre } 2 \text { y } 7 \\
\text { días después de la sesión } \\
\text { final. }\end{array}$ & $\begin{array}{l}\text { Analizar si un programa de } \\
\text { entrenamiento psicomotor } \\
\text { en forma de ejercicio es } \\
\text { capaz de provocar cambios } \\
\text { positivos en las habilidades } \\
\text { de comunicación y } \\
\text { comportamiento de un niño } \\
\text { conTEA. }\end{array}$ & $\begin{array}{l}\text { The Bruininks- } \\
\text { O seretskyTest of } \\
\text { Motor Proficiency } \\
\text { (BOTMP) }\end{array}$ & $\begin{array}{l}\text { BOTM P: } 8 \text { subescalas para } \\
\text { evaluar la motricidad } \\
\text { gruesay y fina. En este } \\
\text { estudio se avalían la } \\
\text { coordinación y el } \\
\text { equilibrio. }\end{array}$ & $\begin{array}{l}\text { Al principio no había } \\
\text { muchas diferencias en } \\
\text { ambos grupos. Tras el } \\
\text { programa de SPARK, se } \\
\text { encontraron diferencias } \\
\text { en el equilibrio estático y } \\
\text { dinámico. El grupo de } \\
\text { tratamiento mostró } \\
\text { mejoría en el equilibrio } \\
\text { estático y dinámico y en } \\
\text { la coordinación bilateral, } \\
\text { en comparación con el } \\
\text { grupo de control. }\end{array}$ & $\begin{array}{l}\text { Tamaños de muestra } \\
\text { relativamente pequeños; } \\
\text { larga duración de la } \\
\text { intervención; diferentes } \\
\text { niveles de motivación en } \\
\text { los participantes conTEA; } \\
\text { variedad en cuanto a la } \\
\text { dedicación de las } \\
\text { actividades físicas. }\end{array}$ \\
\hline $\begin{array}{l}\text { Sarabzadeh, } \\
\text { Bordbar - } \\
\text { Azari, \& } \\
\text { Helalizadeh, } \\
2019 .\end{array}$ & $\begin{array}{l}\text { The effect of } \\
\text { six weeks of } \\
\text { Tai Chai } \\
\text { Chuan } \\
\text { training on } \\
\text { the motor } \\
\text { skills of } \\
\text { children } \\
\text { with Autism } \\
\text { Spectrum } \\
\text { Disorder. }\end{array}$ & $\begin{array}{l}18 \text { niños conTEA } \\
\text { entre } 6 \text { y } 12 \text { años. }\end{array}$ & $\begin{array}{l}2 \text { grupos: el } \\
\text { experimental que } \\
\text { participó durante } 6 \\
\text { semanas en el programa } \\
\text { Tai Chai Chuan, con } 18 \\
\text { sesiones de } 60 \text { min., } 3 \\
\text { días a la semana; y el de } \\
\text { control que no realizó } \\
\text { nada. La M ABC-2 se } \\
\text { aplicó al inicio y al final } \\
\text { del programa. }\end{array}$ & $\begin{array}{l}\text { Investigar la efectividad de } \\
\text { las formas básicas deTai Chi } \\
\text { Chuan para mejorar los } \\
\text { problemas de la función } \\
\text { motora en niños conTEA. }\end{array}$ & $\begin{array}{l}\text { Movement } \\
\text { A ssessment Battery } \\
\text { for Children-2 } \\
\text { (MABC-2) }\end{array}$ & $\begin{array}{l}\text { La batería MABC-2 se } \\
\text { divide en tres rangos de } \\
\text { edad y las tareas, en tres } \\
\text { áreas específicas: destreza } \\
\text { manual, puntería y atrape y } \\
\text { equilibrio estático y } \\
\text { dinámico. }\end{array}$ & $\begin{array}{l}\text { Se observaron diferencias } \\
\text { entre los grupos en las } \\
\text { habilidades de pelota y el } \\
\text { equilibrio. Hubo notables } \\
\text { / diferencias en las } \\
\text { puntuaciones pretest y } \\
\text { postest en las tareas con } \\
\text { balón y equilibrio en el } \\
\text { grupo experimental. }\end{array}$ & $\begin{array}{l}\text { Pequeño tamaño de la } \\
\text { muestra; ausencia de niños } \\
\text { con dificultades cognitivas } \\
0 \text { intelectuales; ausencia } \\
\text { de la evaluación de un } \\
\text { activo estilo de vida. }\end{array}$ \\
\hline $\begin{array}{l}\text { Sumner, } \\
\text { Leonard, \& } \\
\text { Hill, } 2016 .\end{array}$ & $\begin{array}{l}\text { Overlapping } \\
\text { Phenotypes } \\
\text { in Autism } \\
\text { Spectrum } \\
\text { Disorder and } \\
\text { Developmen } \\
\text { tal } \\
\text { Coordination } \\
\text { Disorder: A } \\
\text { Cross- } \\
\text { Syndrome } \\
\text { Comparison } \\
\text { of Motor and } \\
\text { Social Skills. }\end{array}$ & $\begin{array}{l}30 \text { niños conTEA } \\
\text { ( } 25 \text { niños y } 2 \\
\text { niñas) y } 30 \text { niños } \\
\text { con trastorno de } \\
\text { coordinación } \\
\text { (DCD) (21 niños } \\
\text { y } 9 \text { niñas), de } \\
\text { entre } 7 \text { y } 10 \text { años, } \\
\text { fueron } \\
\text { comparados con } \\
35 \text { niños con } \\
\text { desarrollo típico. }\end{array}$ & $\begin{array}{l}\text { Los niños conTEA } \\
\text { completaron las tareas } \\
\text { durante tres sesiones, } \\
\text { que tuvieron lugar en el } \\
\text { laboratorio, durante la } \\
\text { visita al hogar o bien en } \\
\text { su escuela. Los niños con } \\
\text { DCD realizaron tareas } \\
\text { en una o dos sesiones en } \\
\text { el laboratorio o en su } \\
\text { casa. El último grupo se } \\
\text { estudió en su escuela en } \\
\text { dos sesiones. }\end{array}$ & $\begin{array}{l}\text { Comprar el grado de } \\
\text { superposición de las } \\
\text { habilidades motoras y } \\
\text { sociales de los niños con } \\
\text { TEA y niños con trastorno } \\
\text { de coordinación, con niños } \\
\text { n de controles de desar rollo } \\
\text { típico. }\end{array}$ & $\begin{array}{l}\text { Motor milestones } \\
\text { questionnaire } \\
\text { Vineland Adaptative } \\
\text { Behavior Scales } \\
\text { Q uestionnaire II } \\
\text { (VABS-II) } \\
\text { Movement } \\
\text { Assessment Battery } \\
\text { for Children-2 } \\
\text { (MABC-2) }\end{array}$ & $\begin{array}{l}\text { Con los cuestionarios se } \\
\text { mide la motricidad } \\
\text { temprana y actual de los } \\
\text { niños. } \\
\text { M ABC-2: segunda franja } \\
\text { de edad (7-10): destreza } \\
\text { manual, apuntar y atrapar y } \\
\text { equilibrio estático y } \\
\text { dinámico. }\end{array}$ & $\begin{array}{l}\text { Los niños conTEA y } \\
\text { DCD al canzaron más } \\
\text { tarde los hitos motores. } \\
\text { El grupo conTEA } \\
\text { cumplió al límite las } \\
\text { dificulta-des motoras en } \\
\text { / el MABC-2. Además, } \\
\text { alcanzaron niveles más } \\
\text { bajos que los niños con } \\
\text { DT en la evaluación } \\
\text { motora y fueron } \\
\text { calificados como } \\
\text { similares al grupo DCD. }\end{array}$ & $\begin{array}{l}\text { Pequeño número de } \\
\text { participantes; dificultades } \\
\text { de al gunos padres para } \\
\text { recordar el momento en } \\
\text { el que el niño completó } \\
\text { un hitó motor; uso de } \\
\text { escalas del mismo } \\
\text { cuestionario para padres: } \\
\text { dominios motores y de } \\
\text { socialización deVABS. }\end{array}$ \\
\hline
\end{tabular}

for Children-2 (MABC-2), Peabody Developmen-tal M otor Scales-2 (PDM S-2), Test of G ross M otor Development-2 (TGMD-2), Test of Gross Motor Development-3 (TGMD-3), y finalmente la subprueba de coordinación bilateral del SIPT (SIPT-BMC).

Respecto a la traducción y validación para ser aplicadas a la población española, se ha encontrado que las baterías MABC-2, TGMD-2 y TGMD-3 cuentan con versión en castellano. La MABC-2 ha sido adaptada y validad para la población española por Henderson, Sugden, \& Barnett (2012) con una muestra de niños y adolescentes de entre 4 y 16 años. Por su parte, Ayán et al. (2019), tras una intervención con niños españoles de 7 a 10 años, concluyen que, la versión en español de la bateríaTGMD-2 es fiable al objeto de valorar el desa rrollo motor. Finalmente, Estevan, Molina-García, Q ueralt, Álvarez, Castillo, \& Barnett, (2017), tras un proceso para la validación y fiabilidad de la versión en español del TGMD-3, ponen de manifiesto que es adecuada para estudiar el nivel real de competencia motora de los niños en términos de habilidades locomotoras y con el balón y también en términos de habilidades fundamentales de movimiento

Tras el análisis de los artículos seleccionados, se observaron ciertos aspectos que pueden ser determinantes a la hora de elegir las herramientas más adecuadas para implementar en el contexto escolar. Algunas baterías como BOT-2, MABC-2 o TGMD-2, fueron poco empleadas en este ámbito a consecuencia del coste económico que implican (Ruiz-Pérez, Rioja-Collado, Graupera-Sanz, Palomo-Nieto, \& García-Coll, 2015; Cenizo-Benjumea, Ravelo-Afonso, M orilla-Pineda, \& Fernández-Truan, 2017), lo que puede constituir una reducción de las posibilidades de su aplicación.

O tro aspecto que puede condicionar el uso de estos instrumentos es la necesidad de contar con la presencia de una o varias personas durante su aplicación (Rey, Carballo-Fazanes, Varela-Casal, \& A belairas-Gómez, 2020), máxime si se trata de alumnado con TEA. Por ello, es importante que cuenten con «experiencia», con «conocimiento profundo de la materia» y con la «capa cidad de identificar interrogantes, recoger y analizar 
sistemáticamente los datos» (M uñoz-Cantero, LosadaPuente, \& Espiñeira-Bellón, 2015, p. 67).

Por otra parte, cada una de estas baterías o test cuentan con ciertas peculiaridades que pueden actuar como inconvenientes para su puesta en práctica. En primer lugar, se destaca la prueba BOT-2, la cual es muy apropiada y confiable para obtener datos de las habilidades motoras, gruesas y finas, para niños de entre cuatro y 21 años, pues cuenta con ocho subpruebas, cuatro para las destrezas gruesas y cuatro para las destrezas finas, así como cuatro compuestos motores, que incluye a ambas (El Shemy \& El-Sayed, 2018). Se desarrolla de manera individual y su tiempo de aplicación oscila entre 45 y 60 minutos (Asaquibay-Cujilema, 2018). Además, cuenta también con una versión corta (BOT-SF), lacual tieneunaduración de unos 15/20 minutos, aproximadamente. Es muy empleada en diferentes lugares del mundo, pues cuenta con una confiabilidad de 0.92 (Asaquibay-Cujilema, 2018). No obstante, Colebourn, Golub-Victor, \& Paez (2017) indican en su estudio que no es un instrumento muy apropiado para llevar a cabo con niños/ as conTEA por las dificultades en los criterios de la prueba y la demanda de habilidades cognitivas.

La bateríaTGM D-2 es otro de los instrumentos utilizados para evaluar la motricidad de los niños de tres a 10 años y en el que se hace uso de dos subpruebas de seis habilidades locomotoras y seis habilidades de control de objetos (Liu, Breslin, \& ElGarhy, 2017). Es una herra mienta cualitativa con un tiempo de aplicación que no abarca más de 30 minutos (Liu, et al., 2017), su «naturaleza observable» hace que pueda ser una «herramienta ideal para los niños/ as con autismo que a menudo tienen un lenguaje 0 habilidades de comunicación limita dos» (Colebourn, et al., 2017, p. 268). Sin embargo, estos investigadores también constatan que, hasta el momento, no existe un predominante análisis en relación con la confiabilidad de esta batería (Colebourn, et al., 2017) y, aunque hay autores como Ayán et al. (2019) que sí defiende su fiabilidad y validez, en su estudio fue administrada únicamente a niños/ as españoles de siete a 10 años, lo que hace necesario indagaciones con muestras más amplias para obtener una información más sólida. Además, la bateríaTGMD-2 únicamente mide las habilidades motoras gruesas, lo que hace obviar una parte importante de la motricidad y en la que los niños con TEA también tienen dificultades, las destrezas motoras finas.

De la misma forma ocurre con la prueba de integra ción visomotora Beery (VMI), pues es una herramienta que analiza las habilidades viso-perceptuales y motoras que están vinculadas con los movimientos de los dedos y la mano (Gutiérrez-M elgarejo, \& N euta-García, 2015). M ismo también con la subprueba de coordina ción motora bilateral y paradigma experimental de coordinación motora, pues son técnicas que permiten eva luar, como sus propios nombres indican, la coordinación motora, pero dejan a un margen otras destrezas también muy importantes y necesarias de analizar en el ámbito motor.

Por otra parte, cabe señalar que una de las particularidades más comunes y propias de la población con este diagnóstico, es que necesita actuar en un ambiente estructurado y organizado, por lo que es fundamental el uso de pictogramas o pequeñas imágenes que actúen como medio explicativo de cualquier actividad 0 acción. Estos se caracterizan por ser un medio cada vez más accesible al tratarse de un elemento que permite ayudar a las personas conTEA en el desarrollo de cualquier tarea (Regis-Sansalonis, \& Callejón-Chinchilla, 2015) y ser particularmente útiles para mejorar su participación en las sesiones de educación física (MaravéVivas, Carregui-Ballester, Gil-Gómez, \& Chiva-Bartoll, 2021). Por este motivo, se destaca en positivo el soporte visual al que Allen, Bredero, Van, Ulrich, $\&$ Simons (2017) hacen referencia en su estudio en el que se tra baja con la tercera edición del TGMD y con el cual, Ios niños con TEA obtuvieron mejoras significativas en la evaluación motora (Allen, et al. 2017).

0 tro de los instrumentos destacados para la medición deámbito motor en el autismo es el conocido como PDM S-2. Este está compuesto por seis subescalas, una de ellas destinada a los reflejos y las restantes a la medición de la acción estacionaria, manipulación de objetos, integración visomotora, agarre y locomoción (Liu, et al., 2017). Se aplica individualmente y su duración tiende a oscilar entre los 45 a los 60 minutos (Rebelo, Serrano, Duarte-M endes, Paulo, \& Marinho, 2020). Sin embargo, es una herramienta válida y confiable con la que recopilar información sobre el perfil motor de niños hasta los cinco o seis años (Liu, et al., 2017; Rebelo, et al. , 2020), por lo que su uso se ve limitado exclusiva mente a un pequeño grupo de niños/ as con autismo de todo el rango de edad objeto de esta investigación.

Por último, hay que destacar la batería MABC-2, herramienta que fue empleada con éxito para la medición motora de los TEA. Evalúa las destrezas motoras gruesas y finas en niños/ as de entre tres a 16 años de manera individual y la información se obtiene de manera fiable y efectiva en menos de 30 minutos (Liu, et al., 2017), aunque losniños/ as conTEA deben emplear más 
tiempo. Consta de una prueba estandarizada y de una Lista de 0 bservación Conductual (Capistrano, Pinheiro, Portes, Silva, \& Luiz, 2015). La primera está formada por diferentes actividades motoras agrupadas en tres dimensiones (destreza manual, habilidades de puntería y atrape, y equilibrio dinámico y estático), cuyas tareas se orientan de forma diferente en función del grupo de edad con el que se trabaje: niños/ as de tres a seis años, niños/ as de siete a 10 años y niños/ as con 11-16 años (Capistrano, et al., 2015; Liu, et al., 2017; Sarabzadeh, Bordbar-Azari, \& Helalizadeh, 2019). Esta parte de la batería se caracteriza por trabajar con paradigmas cuantitativos y cual itativos, pues además de las puntuaciones obtenidas en cada prueba, se pueden realizar descripciones sobre la manera en la que el niño/ a desarrolla cada tarea (Ruiz-Pérez \& Graupera-Sanz, 2012). Así mismo, también dispone de una Lista de 0 bservación Conductual, instrumento conformado por un cuestionario con diversas preguntas para evaluar el comporta miento motor de los niños de cinco a 12 años en situaciones cotidianas en casa y en la escuela y el cual puede ser aplicado por profesionales (maestros/ as, terapeutas, etc. ) o por losprogenitores (Capistrano, et al. , 2015; Ruiz-Pérez \& Graupera-Sanz, 2012), lo que permitiría obtener información más completa y variada de las destrezas motoras.

\section{Cuestionarios}

Los cuestionarios empleados en uno de los estudios de esta revisión fueron completados únicamente por los familiares y permitieron obtener información sobre las habilidades motoras tempranas y actuales de los niños/ as con TEA. Para ello, se utilizó, por un lado, un cuestionario de hitos motores con el que se puede conocer la edad en la que el niño/ a logró determinadas actuaciones motrices. Sin embargo, tal y como señalan Sumner, Leonard, \& Hill (2016), recoge información de actuaciones que ocurrieron en un pasado, por lo que los datos recogidos pueden ser incompletos.

Por otra parte, el otro instrumento utilizado en este estudio es el conocido como el cuestionario de las Esca las del Comportamiento Adaptativo de Vineland II (VABS-II). A través de él se puede obtener información sobre las habilidades actuales, entre las que se encuentran las relacionadas con la motricidad, en este caso sobre la marcha. Para ello, se utilizan escal as de motricidad gruesa y fina y los familiares indican bajo los términos de «nunca», «a veces» 0 «normalmente» con qué frecuencia se produce esa conducta.
Esta herramienta se caracteriza por evaluar cuatro ámbitos (comunicación, socialización, habilidades para la vida diaria y habilidades motoras) (Soares, \& Cavalcante-N eto, 2015). Por lo contrario, al tratarse de un instrumento que no determina exclusivamente el comportamiento motor ni tampoco está dirigido únicamente a losTEA, el ámbito motriz es estudiado de una manera más superficial (Soares \& Caval cante-N eto, 2015).

Lloyd et al. (2011) y M anwaring, M ead, Swineford, $\&$ Thurm (2017), ponen en valor en sus investigaciones los cuestionarios aplicados a familiares de los sujetos objeto de estudio, pues para obtener una información completa de los niños y, en este caso concretamente, sobre su funcionamiento motor, esunainformación complementaria a pruebas o test, de mucha utilidad. No obstante, estos informes están expuestos a equivocaciones y su fiabilidad puede verse influenciada como consecuencia de «la variabilidad del observador, la variabilidad de lo observado y la variabilidad del cuestionario» (García-Corpas, Esquivel-Prados, \& Pareja-Martínez, 2014, p.47).

\section{Limitaciones y futuras líneas de investiga- ción}

La presente revisión sistemática cuenta con una serie de limitaciones. En primer lugar, cabe destacar que la búsqueda de los estudios se real izó únicamente en las bases de datos mencionadas, pudiendo haber quedado información relevante sin analizar. Además, se estableció un filtro por idioma y año de publicación, seleccionando únicamente aquellas publicaciones en lengua castellana o inglés publicadas entre 2015 y 2020, por lo que los documentos escritos en otras lenguas y con anterioridad a 2015, aunque pudieran ser de interés, también fueron excluidos.

Por último, es necesario señalar que la revisión sistemática realizada tiene carácter cualitativo, es decir, es una revisión sin metaanálisis y que, por lo tanto, se ha presentado la evidencia de forma descriptiva y sin aná lisis estadístico.

Se consideranecesario continuar investigando en esta línea con el fin de poder evaluar el punto de partida e implementar intervenciones en el ámbito escolar para la mejora de las habilidades motoras y el incremento de la práctica físico-deportiva en niños con TEA. También podría ser de interés una línea de investigación específica con el profesorado de educación física de los centros de educación especial y centros ordinarios para 
conocer su percepción sobre la motricidad y AF del alumnado con TEA de sus centros, necesidades del alumnado, necesidades de los centros, necesidades del profesorado, etc. Se podría establecer una estrategia para la evaluación de la motricidad de este alumnado, diseñar acciones de formación e información para el profesorado, así como el establecimiento de un catálogo de actividades físicas escolares/ extraescolares para la mejora de la motricidad y el incremento del nivel de AF de niños conTEA.

\section{Conclusión y aplicaciones prácticas}

Las baterías o test fueron los instrumentos más utilizados para medir la motricidad de los niños conTEA. Los resultados de esta revisión sistemática indican que, aunque la Batería MABC-2 puede ser la recomendada para niños conTEA en el grupo de edad objeto de estudio, existen otros instrumentos que también pueden ser utilizados.

Entre las virtudes de la Batería MABC-2 destaca que las actividades que se proponen para trabajar las diferentes destrezas se dividen en tres grupos de edad, por lo que están adaptadas a las necesidades y potencia lidades de cada uno de ellos, pudiendo ser modificadosy adaptados siempre que sea necesario. Por otra parte, también cuenta con una Lista de O bservación, un tipo de cuestionario con el que se recogen datos de conductas motoras en la vida cotidiana y el cual también puede ser aplicado por los docentes o por los padres y madres.

Sin embargo, y pese a considerarse el instrumento más completo para la evaluación motora de los TEA entre los analizados en este estudio, el coste económico que puede suponer esta Batería es un elemento a tener en cuenta para su puesta en práctica en el contexto escolar. Además, un aspecto a mejorar de esta herramienta puede ser la aplicación de pictogramas con los que poder explicar y estructurar las tareas, pues es primordial para trabajar con estos pequeños teniendo en cuenta las dificultades que presentan en el ámbito comunicativo y organizativo.

EI TEA conlleva dificultades a nivel motor que implica a su vez inactividad física y sedentarismo en las personas diagnósticas, con la consecuente probabilidad de sufrir enfermedades crónicas no transmisibles.

Esta investigación puede servir de ayuda a docentes y profesionales de la AF y el deporte para la selección de instrumentos de evaluación de la motricidad en niños conTEA de 5 a 12 años, como paso previo a posteriores intervenciones con este colectivo para mejorar su salud y calidad de vida. A mayor competencia motriz, más posibilidades de poder realizar actividades físico-deportivas, lo quetambién podríacontribuir a practicar al guna de ellas de forma inclusiva con sus pares sin TEA.

\section{Referencias}

A barca-Sos, A., M urillo-Pardo, B. , Julián-Clemente, J.A. , Zaragoza Casterad, J. \& Generelo-Lanaspa, E. (2015). La educación Física: ¿U naoportunidad parala promoción de la actividad física? Retos: nuevastendencias en Educación Física, deportey recreación, (28), 155-159. https:/ / doi.org/ 10.47197/ retos. v0i28.34946

Adler, B.A., Minshawi, N.F. \& Erickson, C.A. (2014). Evolution of autism: From Kanner to the DSM-V. Handbook of early intervention for autism spectrum disorders: Research, policy and practice, 3-25. DOI:10.1007/ 978-1-49390401-3_1. https:/ / doi.org/ 10.1007/ 978-1-49390401-3_1

Allen, K.A., Bredero, B., Van Damme, T., Ulrich, D.A. \& Simons, J. (2017). Test of Gross M otor D evelopment-3 (TGMD-3) with the U se ofV isual Supportsfor Children withAutism Spectrum D isorder:Validity and Reliability. J Autism Dev Disord, 47(3), 813-833. https:/ / doi.org/ 10.1007/ s10803-016-3005-0

American PsychiatricAssociation (2014). Manual diagnóstico y estadístico de los trastornos mentales (DSM -5), 5a Ed. Madrid, España: Editorial MédicaPanamericana.

Aragunde-Rodríguez, P., Rodríguez-Fernández, J.E., Fontenla Fariña, E. , Gutiérrez-Sas, L. \& Pazos-Couto, J.M . (2018). Autismo y rol del docente en el aula de motricidad en educación infantil. EmásF: R evista Digital deEducación Física, (53), 65-81.

Asaquibay-Cujilema, S. K. (2018). La evaluación clínica infantil: aspectos a considerar en el proceso psicodiagnóstico. Examen complexivo. M achala: UnidadA cadémica de CienciasSociales, Carrerade PsicologíaClínica. Recuperado de: http:/ / repositorio. utmachala. edu.ec/ handle/ 48000/ 12895

Astorino, F., Contini, L., Fessia, G. \& Manni, D. (2018). Efectos de laaplicación de un programa de intervención educativasobrelas habilidades motoras gruesas en individuos con autismo. M HSalud, 15(1), 1-11. https: / / doi.org/ 10.15359/mhs. 15-1.3

Ayán, C., Cancela, J.M., Sánchez-Lastra, M.A., CarballoRoales, A.I., Domínguez-M eis, F. \& Redondo-Gutiérrez, L. (2019). Fiabilidad y validez de la BateríaT GMD-2 en Población Española. Revista Iberoamericana de Diagnóstico y Evaluación, 50, 21-34. https:// doi.org/ 10.21865/ ridep50.1.02

Bishop, M.R. (2014). Motor. En Doreen Granpeesheh e 
JonathanTarbox (ed). Evidence-BasedTreatment for Children with Autism (261-272). 0 xford, UK: Elsevier.

Capistrano, R., Pinheiro Ferrari, E., PortesdeSouza, L., Silva Beltrame, T. \& Luiz Cardoso, F. (2015). Concurrent validation of the MABC-2 M otor Tests and MABC-2 Checklist according to the Developmental Coordination Disorder Q uestionnaire-BR. M otriz: Revista de Educaçao Físca, 21(1), 100-106. https:/ / doi. org/ 10.1590/ s198065742015000100013

Cenizo-Benjumea, J.M ., Ravelo-Afonso, J., M orilla-Pineda, S. \& Fernández-Truan, J.C. (2017).Test de coordinación motriz 3JS: Cómo val orar y analizar su ejecución. Retos, (32), 189-193. https:// doi.org/ 10.47197/ retos. voi32.52720

Colebourn J.A., Golub-Victor, A.C. \& Paez, A. (2017). Developing 0 verhandThrowing Skills for a ChildW ith Autism W ith a CollaborativeA pproach in School-Based Theraphy. Pediatric Physical Therapy, 29 (3), 262-269. https:/ / doi.org/ 10.1097/ pep. 0000000000000405

De Clercq, B., Aelterman, N., De Pauw, S., De Bolle, M., Decuyper, M.\& Tackett, J.L. (2010). Delineating Childhood Autism Spectrum Symptoms from a MaladaptiveTrait Perspective. Journal of Psychopathology and Behavioral Assessment, 32(4), 529-536. https:/ / doi. org/ 10.1007/ s10862-010-9191-8

El Shemy, S.A. \& El-Sayed, M.S. (2018).T Theimpact of auditory rhythmic cueing on gross motor skills in children with autism. The Journal of Physical Therapy Science, 30 (8), 10631068. https:/ / doi.org/ 10.1589/ jpts.30.1063

Estevan, I., MolinaGarcía, J., Queralt,A., Álvarez, O., CastiIlo, I. \& Barnett, L. (2017). Validity and Reliability of the SpanishVersion of theTest of G ross M otor Development3. Journal of M otor Learning and D evelopment, 5(1), 69-81. https:/ / doi. org/ 10.1123/ jmld. 2016-0045

Fuentes, J. , Hervás, A. \& H owlin, P. (2020). ESCAP practice guidance for autism: a summary of evidence based recommendationsfor diagnosisand treatment. European Child \& Adolescent Psychiatry, 1-24. https:/ / doi.org/ 10.1007/ s00787-020-01587-4

García-Corpas, J.P., Esquivel-Prados, E. \& Pareja-Martínez, E. (2014). Fiabilidad de los cuestionarios utilizados en ciencias de la salud. Ars Pharmaceutica, 55 (3), 45-48.

García-M arín, P. \& Fernández-López, N. (2020).Asociación delacompetenciaen las habilidades motrices básicascon las actividadesfísico-deportivas extracurriculares y el índice de masa corporal en preescolares. Retos, 38, 33-39. https:/ / doi.org/ 10.47197/ retos. v38i38.71896

García-Tabuenca, P. (2016). Trastorno del espectro autista (TEA). Anuario del Centro dela Universidad N acional deEducación a Distancia en Calatayud (UNED), (22), 149-162. https:/ / doi.org/ 10.35376/ 10324/ 35103

Gutiérrez-M elgarejo, D.I. \& NeutaGarcía, K.A. (2015). Pre valencia de las habilidades perceptuales visuales, la integra- ción viso-motora, losmovimientos sacádicos, la atención visual y el proceso de lecto-escritura en niñosentre 6-7 años dela ciudad deBogotá en estratos 5 y 6 . Trabajo de M aestríaen Ciencias de laVisión. Bogotá: U niversidad delaSalle, Facultad de Ciencias de la Salud. Recuperado de: https:/ / ciencia. lasalle. edu.co/ maest_ciencias vision/ 36

Henderson, S. E., Sugden, D. y Barnett, L. (2012). Batería de evaluación del movimiento para niños-2 (M ABC-2). Madrid, España: Pearson.

Kamal Nor, N., Hanim Ghozall, A. \& Ismail, J. (2019). Prevalence of $O$ verweight and $O$ besity A mong Children and Adolescents W ith Autism Spectrum Disorder and Associated Risk Factor. Frontiersin Pediatrics, 7(38), 1- 10. https: / / doi.org/ 10.3389/ fped. 2019.00038

Kaur, M., Srinivasan, S. M . \& Bhat, A.N. (2018). Comparing motor performance, praxis, coordination, and interpersonal synchrony between children with and without Autism Spectrum Disorder (ASD). Research in Developmental Disabilities, 72, 79-95. https:/ / doi.org/ 10.1016/ j.ridd.2017.10.025

Kruger, G. R. , Silveira, J. R. \& M arques, A.C. (2019). Motor skills of children with autism spectrum disorder. Rev. Bras. Cineantropom D esempenho H um, 21, 1-8. https: / / doi.org/ 10.1590/ 1980-0037.2019v21e60515

Latorre-Román, P.A., Sánchez-Sal vador, M., Salas-Sánchez, J. $\&$ García-Pinillos, F. (2019). Low level of physical fitness isan early feature in preschool children with autism, Retos, 35, 348-350. https:// doi. org/ 10.47197/ retos. v0i35. 58052

Liberati,A., Altman, D. G., Tetzlaff, J., Mulrow, C., Gøtzsche, P. C., loannidis, F. P., ... \& M oher, D. (2009). The PRISMA statement for reporting systematic reviews and metaanalyses of studies that eval uate health care inter ventions: explanation and elaboration. PLOS M edicine, 6(7), el000100. https:// doi.org/ 10.1371/ journal. pmed. 1000100

Liu, T. (2013). Sensory processing and motor skill performance in elementary school children with autism spectrum disorder. Perceptual and M otor Skills, 116 (1), 197209. https:/ / doi.org/ 10.2466/ 10.25. pms.116.1.197209

Liu, T., Breslin, C.M. \& ElGarhy, S. (2017). Motor skill assessment in Autism Spectrum D isorder: A case study. Physical Educator, 74(2), 239-254. https:/ / doi.org/ 10.18666/ tpe-2017-v74-i2-7148

Lloyd, M., M acD onald, M. \& Lord, C. (2011). M otor skills of toddlerswith autism spectrum disorders. Autism, 17(2), 133-146. https:/ / doi.org/ 10.1177/ 1362361311402230

López-Díaz, J.M ., M oreno-Rodríguez, R. \& López-Bastías, J.L. (2021). Análisis del impacto de un programadeportivo en niños con Trastorno del Espectro del Autismo, Retos, 39, 98-105. https:// doi.org/ 10.47197/ 
retos. v0i39.74841

Mache, M.A. \& Todd, T.A. (2016). Gross motor skills are related to postural stability and age in children with autism spectrum disorder. Research in Autism Spectrum Disorders, 23, 179-187. https:// doi.org/ 10.1016/ j.rasd. 2016.01 .001

Manwaring S.S., M ead D.L., Swineford, L. \& Thrum, A. (2017). Modelling gesture use and early language development in autism spectrum disorder. International Journal of Language\& Communication Disorders, 52 (5), 637651. https:/ / doi. org/ 10.1111/ 1460-6984.12308

Mayes, S.D., Breaux, R.P., Calhoun, S. L. \& Frye, S.S. (2017). High Preval ence of Dysgraphia in ElementaryThrough High School StudentsW ithADHD and Autism. Journal of Attention Disorders, 23(8), 787-796. https:/ / doi.org/ $10.1177 / 1087054717720721$

Muñoz-Cantero, J.M., Losada-Puente, L. \& Espiñeira-Bellón, E.M. (2015). Perfil y competencias del profesional como evaluador en instituciones de atención a personas con discapacidad intelectual. Siglo Cero, 46(4), 59-74. https:/ / doi.org/ 10.14201/ scero20154645974

Najafabadi, M.G., Sheikh, M., Hemayattalab, R., M emari,A., Aderyani, M . R. \& H afizi, S. (2018). The effect of SPARK on social and motor skills of children with autism. Pediatrics and Neonatology, 59(5), 481-487. https:/ / doi.org/ 10.1016/ j. pedneo.2017.12.005

Prieto, J.A., Cueto, S., Carballo-Fazanes, A., \& A belairasGómez, C. (2020). Psychomotor development disorders in apparently healthy children and considerations of family evaluation. Journal of $\mathrm{H}$ uman Sport and Exercise, in press. https:/ / doi.org/ 10.14198/ jhse. 2022.173.04

Rebelo, M, Serrano, J., Duarte-M endes, P., Paulo, R., \& Marinho, D.A. (2020). Desenvolvimento Motor da criança. Relaçao entre Habilidades M otoras $\mathrm{Globais,} \mathrm{Ha}$ bilidades M otorasFinase Idade. Cuadernos de Psicología del Deporte, 20(1), 75-85. https:/ / doi. org/ 10.6018/ cpd. 385791

Regis-Sansalonis, P.J., \& Callejón-Chinchilla, M .D. (2015). Del pictogramaala imagen: herramientas de comunicación y lenguaje en per sonas con síndrome deA sperger a través de recursos visuales para la inclusión social. Arteterapia-Papeles de arteterapia y educación artística para la inclusión social, 10, 329-341. https:/ / doi. org/ 10.5209/ rev arte. 2015. v10.51700

Reinders, N.J. , Branco,A.,W right, K., Fietcher, P.C. \& Bryden, P.J. (2019). Scoping Review: Physical Activity and Social Functioning in Young People W ith Autism Spectrum Disorder. Frontiers in Psychology, 10(120), 1-17. https:/ / doi.org/ 10.3389/ fpsyg. 2019.00120

Rey, E., Carballo-Fazanes, A., Varela-Casal, C. \& A belairasGómez, C. on behalf of ALFA-MOV Project collaborators. (2020). Reliability of the test of grossmotor development: A systematic review. PLOS ON E 15(7): e0236070. https:// doi. org/ 10.1371/ journal. pone. 0236070

Ruiz-Pérez, L.M . \& Graupera-Sanz, J.L. (2012). Batería de evaluación del movimiento para niños-2 (M ABC-2). Madrid, España: Pear son Educación

Ruiz-Pérez, L.M., RiojacCollado, N., Graupera-Sanz, J.L., Pa lomo-N ieto, M . \& García-Coll,V. (2015). Grami-2: Desarrollo de un test para evaluar la coordinación motriz global en la educación primaria. Revista Iberoamericana de Psicología del Ejercicio y el Deporte, 10(1), 103-111. https:/ / doi.org/ 10.5232/ ricyde2017.04907

Ruiz-Vicente, D., Salinero, J.J., González-Millán, C., LledóSoriano, M ., García-Pastor,T.,Theirs, C.I., ... \& Guitián,A. (2015). Descripción de la práctica de actividad física, habilidades motrices básicas y composición corporal en niños y jóvenes de espectro autista. Diferencias de sexo. Retos, 28, 61-65. https:// doi.org/ 10.47197/ retos. v0i28. 34817

Sarabzadeh, M., Bordbar-Azari, B. \& Helalizadeh, M. (2019). The effect of six weeks ofTai $C$ hi Chuan training on the motor skills of children withAutism Spectrum Disorder. Journal of Bodywork and Movement Therapies, 23(2), 284290. https: / / doi.org/ 10.1016/ j.jbmt. 2019.01.007

Soares, A.M. \& Cavalcante-N eto, J.L. (2015). Avaliçao do Comportamento M otor em Crianças comTranstorno do Espectro do Autismo: uma Revisão Sistemática. Revista Brasileira de Educação Especial, 21(3), 445-458. DOI: https:// doi.org/10.1590/s141365382115000300010

Sowa, M. \& Meulenbroek, R. (2012). Effects of physical exercise onAutism Spectrum Disorders: A meta analysis Research in Autism Spectrum Di sorders, 6 (1), 46-57. https:/ / doi.org/ 10.1016/ j.rasd.2011.09.001

Sumner, E., Leonard, H.C.\& Hill, E.L. (2016). 0 verlapping Phenotypes in Autism Spectrum Disorder and Developmental Coordination Disorder: A CrossSyndromeComparison of M otor and Social Skills. Journal of Autism and D evelopmental Disorders, 46 (8), 2609-2620. https: / / doi. org/ 10.1007/ s10803-016-2794-5

M aravé-Vivas, M., Carregui-Ballester, J., Gil-Gómez, J. \& Chiva-Bartoll, 0. (2021). Hacialainclusión del alumnado conTEA en educación física: investigaciónacción en un programa piloto. Retos, 42, 66-76. https:/ / doi.org/ 10.47197/ retos. v42i0.85845

Yu, C C.W., Wong, S.W.L., Lo, F.S.F., So, R. C.H. \& Chan, D.F.Y. (2018). Study protocol: arandomized controlled trial study on the effect of agame-based exercise training program on promoting physical fitness and mental health in children with autism spectrum disorder. BM C Psychiatry, 18(56). https: / / doi.org/ 10.1186/ s12888-018-16359 